\title{
FLEXIBLE MANUFACTURING SYSTEMS MODELLING AND PERFORMANCE EVALUATION USING AUTOMOD
}

\author{
Reddy, B. S. P.* \& Rao, C. S. P.* \\ * Dept. of Mechanical Engg., Kakatiya Institute of Tech. \& Science, Warangal-15, A.P., India \\ ${ }^{* *}$ Dept. of Mechanical Engg., National Institute of Technology, Warangal-4, A.P., India \\ E-Mail: bspr.kits@gmail.com; csprao63@yahoo.com
}

\begin{abstract}
In recent times flexible manufacturing systems emerged as a powerful technology to meet the continuous changing customer demands. Increase in the performance of flexible manufacturing systems is expected as a result of integration of the shop floor activities such as machine and vehicle scheduling. The authors made an attempt to integrate machine and vehicle scheduling with an objective to minimize the makespan using Automod. Automod is a discrete event simulation package used to model and simulate a wide variety of issues in automated manufacturing systems. The key issues related to the design and operation of automated guided vehicles such as flow path layout, number of vehicles and traffic control problems are considered in the study. The performance measures like throughput, machine and vehicle utilization are studied for different job dispatching and vehicle assignment rules in different flexible manufacturing system configurations.

(Received in August 2010, accepted in April 2011. This paper was with the authors 1 month for 2 revisions.)
\end{abstract}

Key Words: Flexible Manufacturing System, Simultaneous Scheduling, Automated Guided Vehicle, Simulation, Automod

\section{INTRODUCTION}

The present day manufacturing environments are highly agile and are confronted with the continuous changing customers' requirements. Flexible Manufacturing Systems (FMS) emerged as a powerful one due to its wide flexibility, which is essential to stay competitive in this highly dynamic environment. FMS is a complex concurrent system consisting elements like machines, automated guided vehicles, storage and retrieval system. FMS scheduling problems are more complex, since the components are highly interrelated and in addition has multiple part types and alternative routings.

Typically, parts in an FMS visit different machines for different operations and thus generate a great demand for vehicles. Automated guided vehicles (AGV) are widely used in FMS due to their flexibility and adaptability. Vehicle scheduling is crucial in FMS for timely transfer of parts between the work centres and the decisions include the design of flow path layout, traffic control and vehicle dispatching rules.

Simulation is a powerful tool used to imitate the system behaviour over time and to draw inferences about its real time performance. Simulation is used to study the various control strategies before one can suggest an optimum solution for the given problem, hence simulation is used as a decision support system for real time scheduling of manufacturing systems and is found to be an effective tool in design and operation of an FMS.

The authors made an attempt to address simultaneously both the machine and vehicle scheduling in a given FMS environment with an objective to minimize the job completion time using Automod a powerful discrete event simulation package. Extensive simulation 
studies are performed on different FMS layouts for different job and vehicle dispatching rules in order to derive an optimum schedule for the given FMS environment.

\section{LITERATURE REVIEW}

Egbelu and Tanchoco proposed different heuristic rules for dispatching automatic guided vehicles in a job shop environment [1]. Mahadevan and Narendran addressed the key issues involved in the design and operation of AGV-based material handling systems for an FMS and analyzed the problems arising from multi-vehicle systems [2]. Raju and Chetty developed priority nets for flexible and realistic modelling and simulation of FMS [3]. Kim and Kim presented a simulation-based real time scheduling methodology for FMS [4]. Drake and Smith described the use of a discrete event simulation for controlling a FMS and used the simulation model for the system design, analysis and control [5]. Ulusoy et al. addressed simultaneous scheduling of machines and AGVs in an FMS for the makespan minimization using genetic algorithms. They randomly assigned a vehicle for each job operation, developed a special crossover operator and used repair function for any violation of the precedence constraints [6].

Sabuncuoglu studied the effect of scheduling rules on the performance of FMS, tested different machine and AGV rules against the mean flow time criterion [7]. Anwar and Nagi considered simultaneous scheduling of AGVs and machines in the production of complex assembled products. A heuristic was used and minimized the times by exploiting the critical path of an integrated operation network [8]. Ozden used simulation to investigate the effect of AGV design factors on the throughput and machine utilizations of an FMS [9]. Rajotia et al. proposed a heuristic methodology for configuring a mixed (hybrid) uni/bi-directional flow path for an AGV material handling system. They conducted simulation studies to compare the productive potentials for uni-directional, mixed (uni/bi-directional), bi-directional flow path design alternatives [10].

Paprotny et al. described a methodology to input automatically the system components to describe an automated material handling system within the simulation language. The method is based on data extraction from a CAD layout file of the system, automatically generates the components and reduces the model building time [11]. Schulz et al. proposed a generic model of a $300 \mathrm{~mm}$ wafer fabrication facility and studied the impact of a given automated material handling system and the interactions between the material flow and the factory performance using Automod [12]. Rohrer focused on material handling and emphasized the importance of Automod in design, analysis and operation of manufacturing systems [13]. Sly and Moorthy discussed the sequential data exchange (SDX) and its implementation for the Automod integration of the factory layout and simulation technologies [14].

White et al. designed and implemented a simulation model of a mail distribution system that communicates with the system hardware controller and used model communication module (MCM) functions in Automod to send data from the simulation model to the system controller [15]. Smith classified the literature on the use of discrete event simulation for manufacturing systems mainly into three primary classes such as manufacturing system design, manufacturing systems operations and simulation package developments for manufacturing systems applications [16]. Reddy and Rao addressed multi-objective optimization of machines and AGVs in an FMS using a hybrid genetic algorithm on different FMS layouts configurations and some of the layouts are considered in the present study [17]. Haijun et al. modelled a general steel logistics centre based on the Automod simulation platform by using simulation technique and then validated the same [18]. Chenglin et al. modelled an integrated logistics park and simulated by application of fuzzy clustering theory and Automod simulation technology [19]. 


\section{FMS MODELLING AND SIMULATION USING AUTOMOD}

Automod is used for modelling and simulation of a wide variety of issues in automated manufacturing systems. The Automod consists of two distinct environments, the edit and simulation environments. In the edit environment the manufacturing system model is built based on the number of machines and the AGV path layout. In the simulation environment code is written to generate loads and to describe the movement procedure and finally the model is run to observe the real time performance behaviour of the manufacturing system. The simulation model facilitates to change the inputs and to study lots of variants of schedules before one can choose the best solution for the given problem.

\subsection{Problem definition}

The Scheduling Problem is described by a finite set $J$ of $n$ jobs $\left\{J_{i}\right\} 1 \leq i \leq n$ and a set $M$ of $m$ machines $\left\{M_{k}\right\} 1 \leq k \leq m$. Each job $J_{i}$ has to be processed on every machine and consists of a chain of $m_{i}$ operations $\left\{O_{i k}\right\} 1 \leq i \leq n, 1 \leq k \leq m_{i}$ which have to be scheduled in a strictly sequential order. $O_{i k}$ denotes the operation of job $J_{i}$ that has to be processed on machine $M_{k}$ for a certain uninterrupted processing time $P_{i k}$. Vehicles move jobs between different machines for different operations based on the machine sequence. $V_{m i k}$ denotes the travelling time from machine $m_{i}$ to machine $m_{k}$ and the job completion times include the travelling and processing times. Representation of operations of a job, objective criteria, job and vehicle dispatching methodologies considered in the present study are discussed below.

\subsection{Representation of operations of a job}

The representation is explained considering an example with 4 machines and 3 jobs. Job 1 consists of 3 operations, job 2 consists of 2 operations and job 3 consists of 2 operations. Total there are 7 operations and 1 represents $1^{\text {st }}$ operation of first job, 4 represents first operation of $2^{\text {nd }}$ job and so on. The precedence constraints in a job shop are crucial and cannot be violated; hence necessary care is taken during the operation schedule generation itself.

\begin{tabular}{lcccccccc} 
No. of job: & & 1 & \multicolumn{3}{c}{2} & \multicolumn{2}{c}{3} \\
Operations on each job: & 1 & 2 & 3 & 1 & 2 & 1 & 2 \\
Machines: & $M_{1}$ & $M_{3}$ & $M_{4}$ & $M_{2}$ & $M_{4}$ & $M_{3}$ & $M_{1}$ \\
Representation: & 1 & 2 & 3 & 4 & 5 & 6 & 7
\end{tabular}

\subsection{Objective criteria}

Makespan referred to as the total completion time of all the jobs is the objective criteria value and is evaluated based on the operation sequence generated.

Operation completion time: $O_{i j}=P_{i j}+T_{i j} \quad\left(i^{\text {th }}\right.$ operation of $j^{\text {th }}$ job $)$

$P_{i j}$ - Processing time, $T_{i j}$ - Travelling time

Job completion time: $C_{j}=\sum_{\mathrm{j}=1}^{\mathrm{n}} O_{i j}$ (Completion time of all operations of the job)

Makespan: $C_{j \max }=\max \left(C_{1}, C_{2}, \ldots C_{n}\right)$ (Maximum completion time of all the jobs)

\subsection{Job dispatching methodology}

Initially all the jobs are available at the load/unload station and the vehicles move jobs to the corresponding machines for processing based on one of the job dispatching rules such as First come first serve (FCFS), Shortest processing time (SPT) and Longest processing time (LPT). 
As soon as the jobs move from one machine to the other, they move into the input buffer and then onto the machine as and when it is available. As and when the operations are completed the jobs move into the output buffer and then onto the AGV for next operation. The jobs are sequenced in chronological order according to FCFS rule, in the increasing order of processing times according to SPT rule and in the decreasing order of the processing times according to LPT rule.

\subsection{Vehicle scheduling methodology}

A scheduling list is a sequence of locations that act as a reference for the vehicles to claim work. When a vehicle is idle it looks at the list for assignment, once a vehicle is assigned a task, it cannot be interrupted or reassigned until the task is complete. As and when a vehicle receives a request from a job, the vehicle moves to the job picks it up and moves to the next machine based on the job operation sequence. The vehicle assignments are made according to one of the three heuristics available such as "At, Closest and Oldest rules". According to the 'At' rule the vehicle first search for work at the corresponding machine, according to the 'Closest' rule the vehicle search for work at the nearest location and according to the 'Oldest' rule the vehicle search for work at the location which completed the work first. If a vehicle fails to find a work it looks at the parking list, claims one unit of parking location and moves to it.

\subsection{Simulation code}

The authors developed a VC++ program to arrange the data of the standard job shop scheduling problem in the form of three text files job sequence, machine sequence and processing times. The job sequence defines the sequence in which the jobs are to be dispatched, machine sequence gives the sequence of machines a job has to visit for its operations and the processing time gives the job operation times on corresponding machines.

Simulation code is developed for load generation and the movement procedure through the system. Loads are generated based on the number of jobs and initially all the loads and vehicles are available at the load/unload station and will be moved to the corresponding machines based on the job sequence. The job movement procedure is to move the job into the input buffer of the machine, after processing move to the output buffer and later to the input buffer of the next machine based on the operation sequence. During the load movement process the vehicles accomplish loading/unloading operations and moving the jobs from load/unload station to machines and from one machine to the other. The simulation code for load generation and movement procedure is given below.

\section{Simulation code for data files, loads and processes:}

\section{Job Sequence}

set vi_opno(vi)=vi_i

read vi_jobno(vi), vi_machineno(vi),vr_comptime(vi) from vf_input

read vi_i from vf_input

inc vi by 1

\section{Machine sequence}

set $\mathrm{vi} \_\mathrm{j}=1$

while vi_j $<=$ vi_NumOpertionsPerJob(vi_i) do begin

read vi_jobseq(vi_i,vi_j) from vf_input

inc vi_j by 1

inc vi_totalop by 1 


\section{Processing Times}

set vi_j $=1$

while vi_j $<=$ vi_NumMachPerJob(vi_i) do begin

read vr_JobCycleTime(vi_i,vi_j) from vf_input

inc vi_j by 1

\section{Load Generation Procedure}

set vloc_Machine $(1)=$ AGV:cp_m1

set vloc_Machine(n) = AGV:cp_mn (n: number of machines)

send to p_loadgen

\section{Load Arriving Procedure}

set ai_i $=1$

while ai_i $<=$ vi_NoofJobs do begin

set ai_opno=vi_opno(ai_i)

set ai_jobno=vi_jobno(ai_i)

set ai_machineno=vi_machineno(ai_i)

clone 1 to p_main

inc ai_i by 1

\section{Load Movement Procedure}

set vit $=0$

move into AGV:cp_lu

set ai_i $=1$

while ai_i $<=$ vi_NumOpertionsPerJob(ai_jobno) do begin

travel to vloc_Machine(ai_machineno)

move into q_in(ai_machineno)

move into q_machine(ai_machineno)

get r_machine(ai_machineno)

wait for vr_JobCycleTime(ai_jobno,ai_machineno) min

free r_machine(ai_machineno)

inc vit by 1

move into q_out(ai_machineno)

move into vloc_Machine(ai_machineno)

set the next machine based on sequence

set ai_machineno $=$ vi_jobseq(ai_jobno,ai_i +1$)$

inc ai_i by 1

\section{Notations used in the simulation code:}

opno - Operation number

jobno - Job number

Machineno - Machine number

Comptime - Completion time

Jobseq - Job sequence

Totalop - Total number of operations

NoofJobs - Number of jobs

NumOperationsPerJob - Total number of operations required per job

NumMachPerJob - Number of machines required per job

JobCycleTime - Job cycle time

$\mathrm{Lu}$ - Load/unload station

in - Input buffer

out - Output buffer

p_loadgen - load generation 


\subsection{Performance analysis of the developed model}

Once the simulation model is ready, run the model to study the behaviour of the manufacturing system under the given set of conditions. The necessary text files can be edited to study the performance of the manufacturing system for different job dispatching and vehicle assignment rules to derive an optimum schedule. The Automod step by step simulation procedure is shown in form of a flow chart in Fig1 and the detailed step by step working procedure of the proposed simulation approach is explained with a numerical illustration.

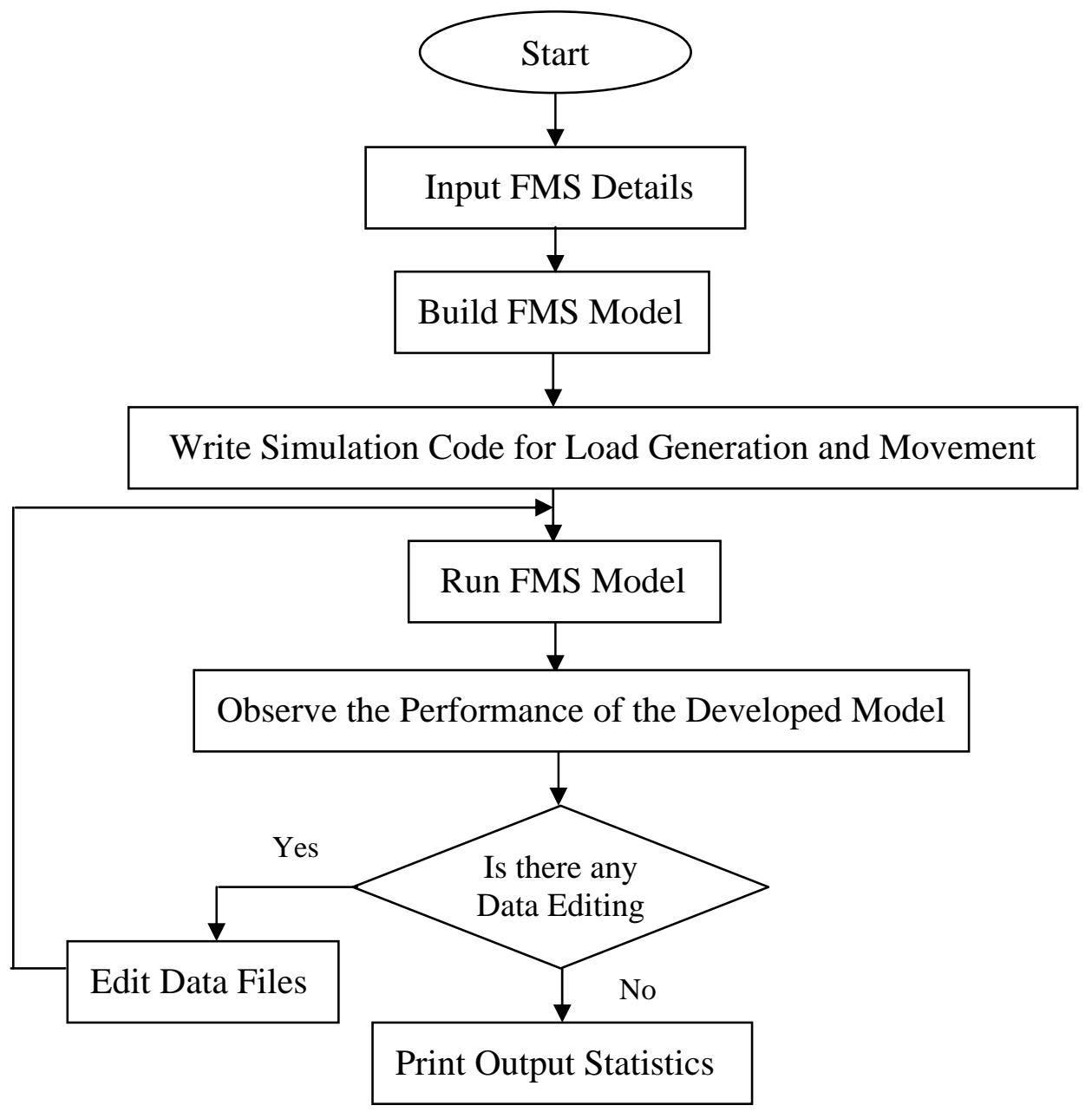

Figure 1: Flow chart of step by step Automod Simulation procedure.

\section{NUMERICAL ILLUSTRATION}

FMS Environment: Consider an FMS environment with 5 machines as shown in Fig. 2 and its corresponding travel times are given in Table I. 10 jobs each with 5 operations are considered and the job set details i.e., processing times of operations of a job, machine sequence of job operations and the sequence of jobs for dispatching are presented in Tables II, III and IV respectively. 2 AGVs are considered to move the jobs between machines for different operations. Vehicles move with a speed of $40 \mathrm{~m} / \mathrm{min}$, loading and unloading times of 30 seconds each are considered. The Automod simulation results regarding the process, traffic, resources and AGV statistics for 'LPT' job dispatching rule and 'At' vehicle assignment rule are presented as output in Tables V, VI, VII and VIII respectively. Finally the comparison of different job and vehicle dispatching rules are shown in Fig. 3. 


\section{INPUT}

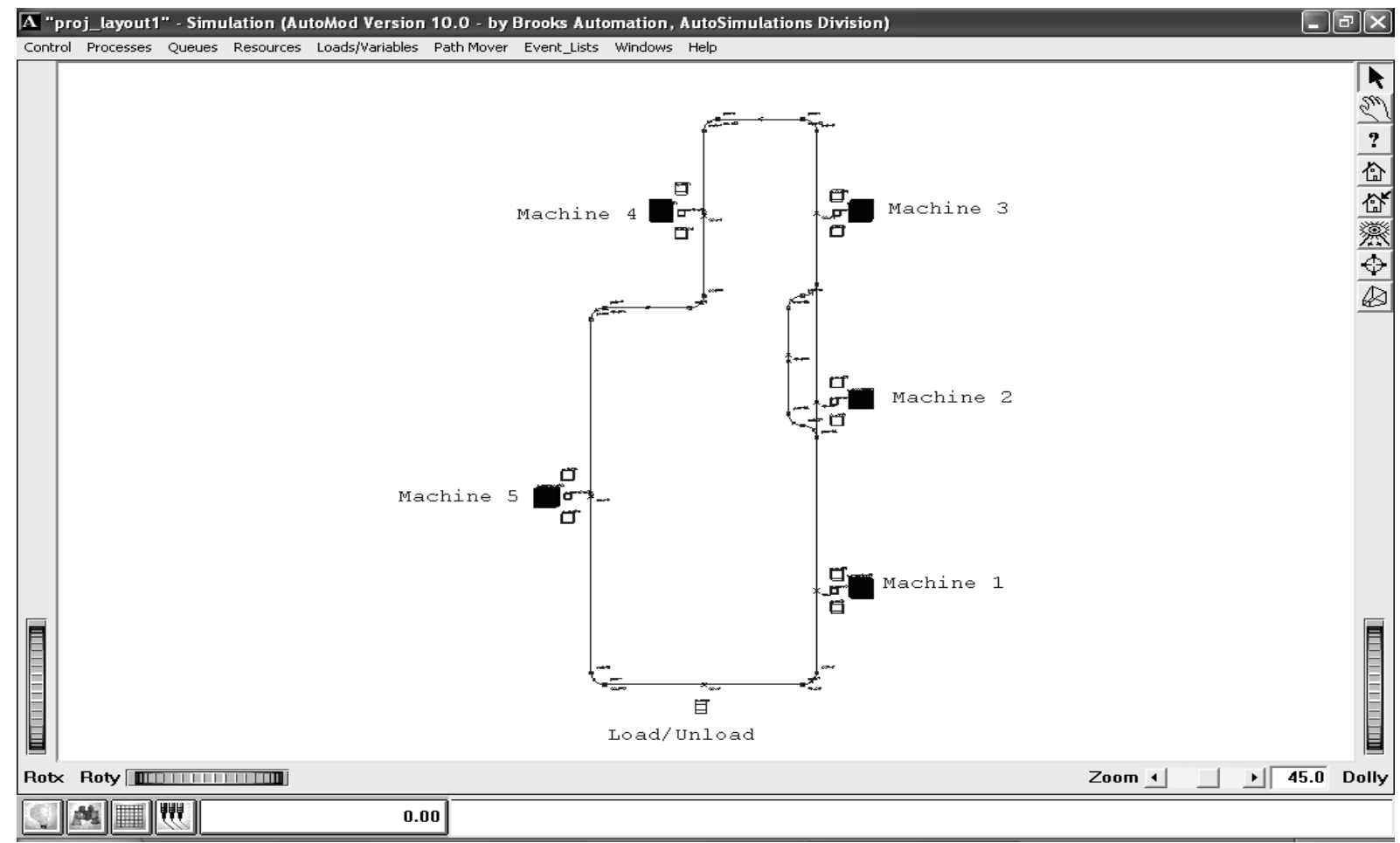

Figure 2: FMS Layout 1.

Table I: Travel time Matrix 1.

\begin{tabular}{|c|c|c|c|c|c|c|}
\hline & $\mathrm{L} / \mathrm{U}$ & $\mathrm{M}_{1}$ & $\mathrm{M}_{2}$ & $\mathrm{M}_{3}$ & $\mathrm{M}_{4}$ & $\mathrm{M}_{5}$ \\
\hline $\mathrm{L} / \mathrm{U}$ & 0 & 3 & 5 & 7 & 9 & 14 \\
\hline $\mathrm{M}_{1}$ & 15 & 0 & 3 & 5 & 8 & 12 \\
\hline $\mathrm{M}_{2}$ & 13 & 15 & 0 & 3 & 6 & 10 \\
\hline $\mathrm{M}_{3}$ & 11 & 13 & 15 & 0 & 4 & 8 \\
\hline $\mathrm{M}_{4}$ & 7 & 9 & 11 & 13 & 0 & 5 \\
\hline $\mathrm{M}_{5}$ & 4 & 6 & 8 & 10 & 13 & 0 \\
\hline
\end{tabular}

Table II: Job operations processing times.

\begin{tabular}{|c|c|c|c|c|c|}
\hline \multirow{2}{*}{ Job No. } & \multicolumn{5}{|c|}{ Processing times } \\
\cline { 2 - 6 } & $\mathrm{M}_{1}$ & $\mathrm{M}_{2}$ & $\mathrm{M}_{3}$ & $\mathrm{M}_{4}$ & $\mathrm{M}_{5}$ \\
\hline 1 & 53 & 21 & 34 & 55 & 95 \\
\hline 2 & 21 & 71 & 26 & 52 & 16 \\
\hline 3 & 12 & 42 & 31 & 39 & 98 \\
\hline 4 & 55 & 77 & 66 & 77 & 79 \\
\hline 5 & 83 & 19 & 64 & 34 & 37 \\
\hline 6 & 92 & 54 & 43 & 62 & 79 \\
\hline 7 & 93 & 87 & 87 & 69 & 77 \\
\hline 8 & 60 & 41 & 38 & 24 & 83 \\
\hline 9 & 44 & 49 & 98 & 17 & 25 \\
\hline 10 & 96 & 75 & 43 & 79 & 77 \\
\hline
\end{tabular}


Table III: Job operations machine sequence.

\begin{tabular}{|c|c|c|c|c|c|}
\hline Job No. & \multicolumn{5}{|c|}{ Machine sequence } \\
\hline 1 & 2 & 1 & 5 & 4 & 3 \\
\hline 2 & 1 & 4 & 5 & 3 & 2 \\
\hline 3 & 4 & 5 & 2 & 3 & 1 \\
\hline 4 & 2 & 1 & 5 & 3 & 4 \\
\hline 5 & 1 & 4 & 3 & 2 & 5 \\
\hline 6 & 2 & 3 & 5 & 1 & 4 \\
\hline 7 & 4 & 5 & 2 & 3 & 1 \\
\hline 8 & 3 & 1 & 2 & 4 & 5 \\
\hline 9 & 4 & 2 & 5 & 1 & 3 \\
\hline 10 & 5 & 4 & 3 & 2 & 1 \\
\hline
\end{tabular}

Table IV: Job sequence.

\begin{tabular}{|c|c|c|}
\hline Operation No. & Job No. & Machine No. \\
\hline 31 & 7 & 4 \\
\hline 46 & 10 & 5 \\
\hline 16 & 4 & 2 \\
\hline 26 & 6 & 2 \\
\hline 1 & 1 & 2 \\
\hline 36 & 8 & 3 \\
\hline 21 & 5 & 1 \\
\hline 41 & 9 & 4 \\
\hline 11 & 3 & 4 \\
\hline 6 & 2 & 1 \\
\hline
\end{tabular}

\section{OUTPUT}

Table V: Process statistics (Number of loads for processing in the system).

\begin{tabular}{|l|c|c|c|c|c|c|c|c|c|}
\hline Time: 8:00:00 & Total & Cur & Average & Capacity & Max & Min & Util & Avg_time & Avg_wait \\
\hline p_init & 1 & 0 & 0.00 & -- & 1 & 0 & -- & 0.00 & -- \\
p_loadgen & 1 & 0 & 0.00 & -- & 1 & 0 & -- & 0.00 & -- \\
p_main & 10 & 10 & 10.00 & -- & 10 & 0 & -- & 28800.00 & -- \\
\hline
\end{tabular}

Table VI: Traffic statistics (Number of loads moving in the system).

\begin{tabular}{|l|c|c|c|c|c|c|c|c|c|}
\hline Time: 8:00:00 & Total & Cur & Average & Capacity & Max & Min & Util & Avg_time & Avg_wait \\
\hline P_init & 1 & 0 & 0.00 & Infinite & 1 & 0 & -- & 0.00 & -- \\
P_loadgen & 1 & 0 & 0.00 & Infinite & 1 & 0 & -- & 0.00 & -- \\
P_main & 10 & 10 & 10.00 & Infinite & 10 & 0 & -- & 28800.00 & -- \\
\hline
\end{tabular}

Table VII: Resource statistics (Machine utilization).

\begin{tabular}{|l|c|c|c|c|c|c|c|c|c|}
\hline Time:8:00:00 & Total & Cur & Avg & Capacity & Max & Min & Util & Avg_time & Avg_wait \\
\hline r_machine(1) & 7 & 1 & 0.81 & 1 & 1 & 0 & 0.81 & 3333.48 & 2980.57 \\
r_machine(2) & 9 & 1 & 0.95 & 1 & 1 & 0 & 0.95 & 3055.61 & 4113.83 \\
r_machine(3) & 7 & 1 & 0.66 & 1 & 1 & 0 & 0.66 & 2713.72 & 356.36 \\
r_machine(4) & 8 & 1 & 0.66 & 1 & 1 & 0 & 0.66 & 2382.34 & 1381.96 \\
r_machine(5) & 8 & 1 & 0.97 & 1 & 1 & 0 & 0.96 & 3479.58 & 3652.11 \\
\hline
\end{tabular}


Table VIII: AGV statistics (AGV utilization).

\begin{tabular}{|c|c|c|c|c|c|c|c|c|c|c|}
\hline 8:00 & \multicolumn{3}{|c|}{ Delivering } & \multicolumn{3}{c|}{ Retrieving } & \multicolumn{3}{c|}{ Going to park } & Parking \\
\hline Name & $\begin{array}{c}\text { Percent } \\
\text { of Total } \\
\text { time }\end{array}$ & $\begin{array}{c}\text { Trips } \\
\text { made }\end{array}$ & $\begin{array}{c}\text { Average } \\
\text { time/trip } \\
\text { (min) }\end{array}$ & $\begin{array}{c}\text { Percent } \\
\text { of Total } \\
\text { time }\end{array}$ & $\begin{array}{c}\text { Trips } \\
\text { Made }\end{array}$ & $\begin{array}{c}\text { Average } \\
\text { time/trip } \\
\text { (min) }\end{array}$ & $\begin{array}{c}\text { Percent } \\
\text { of Total } \\
\text { time }\end{array}$ & $\begin{array}{c}\text { Trips } \\
\text { made }\end{array}$ & $\begin{array}{c}\text { Average } \\
\text { time/trip } \\
\text { (min) }\end{array}$ & $\begin{array}{c}\text { Percent } \\
\text { of Total } \\
\text { time }\end{array}$ \\
\hline AGV1 & 0.369 & 21 & 506.63 & 0.333 & 22 & 435.29 & 0.142 & 8 & 512.19 & 0.156 \\
AGV2 & 0.336 & 21 & 460.64 & 0.295 & 21 & 405.2 & 0.267 & 11 & 699.43 & 0.100 \\
\hline
\end{tabular}

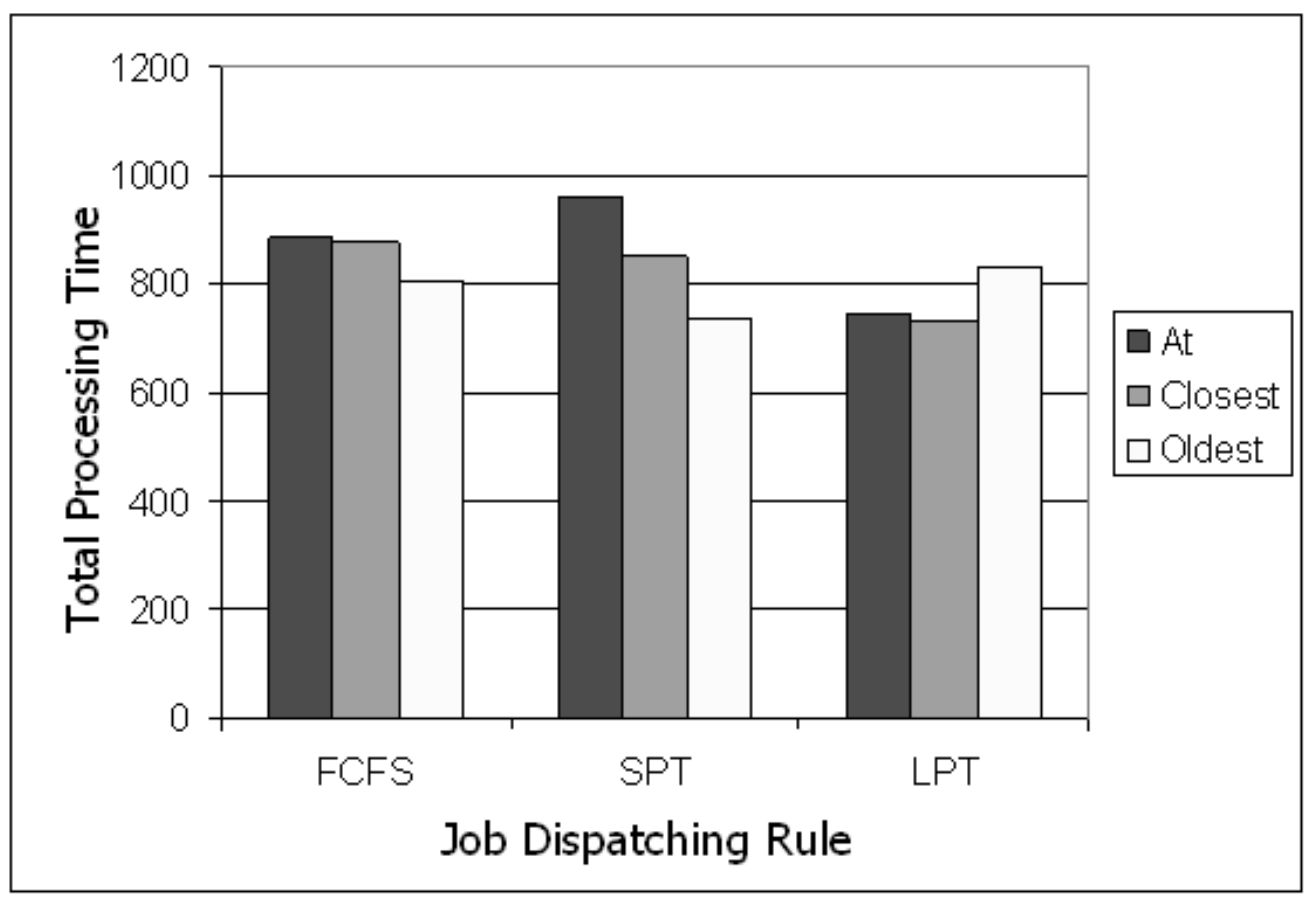

Figure 3: Performance comparison of different job and vehicle dispatching rules.

\section{EXTENSIVE SIMULATION STUDIES}

Extensive simulation studies were performed on different FMS layout model configurations (uni-directional and bi-directional) with different job sets (up to 100 operations) for various job dispatching rules (FCFS, SPT and LPT) and vehicle assignment rules (At, Closest and Oldest). The problem sets are taken from the standard job shop scheduling problems reported in [20] and [21]. A maximum of 100 operations are considered in the study on both uni- and bi-directional layouts. The different FMS layouts considered in the study are shown in Figs. 3 and 4 and their corresponding Travel Times are given in Tables IX and X. The results of the large size problems studied on different FMS environments for different job dispatching and vehicle assignment rules are presented in Table XI, where $L$ indicates the layout number and $J$ indicates the job set number. 
Reddy, Rao: Flexible Manufacturing Systems Modelling and Performance Evaluation ...

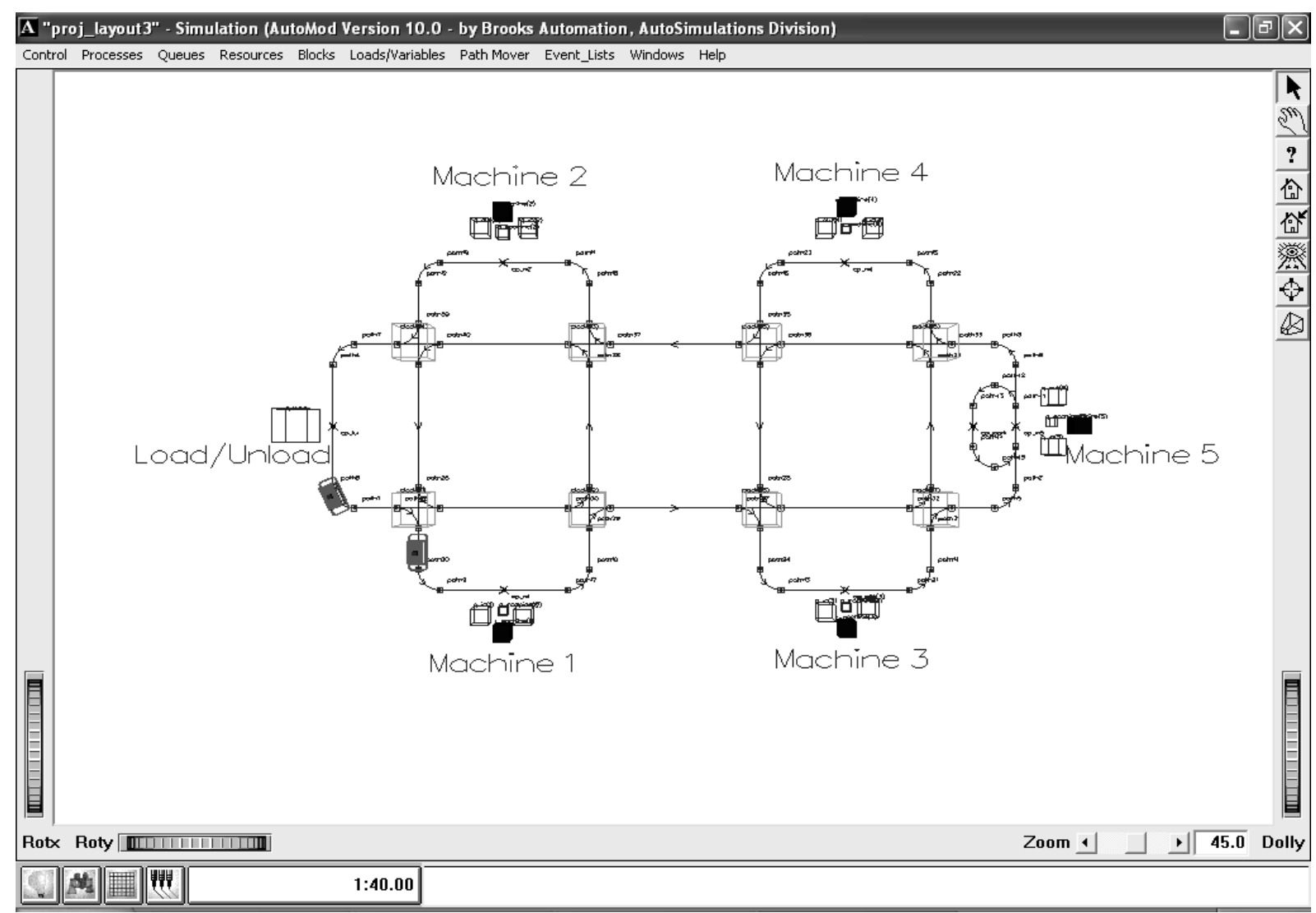

Figure 3: FMS Layout 2.

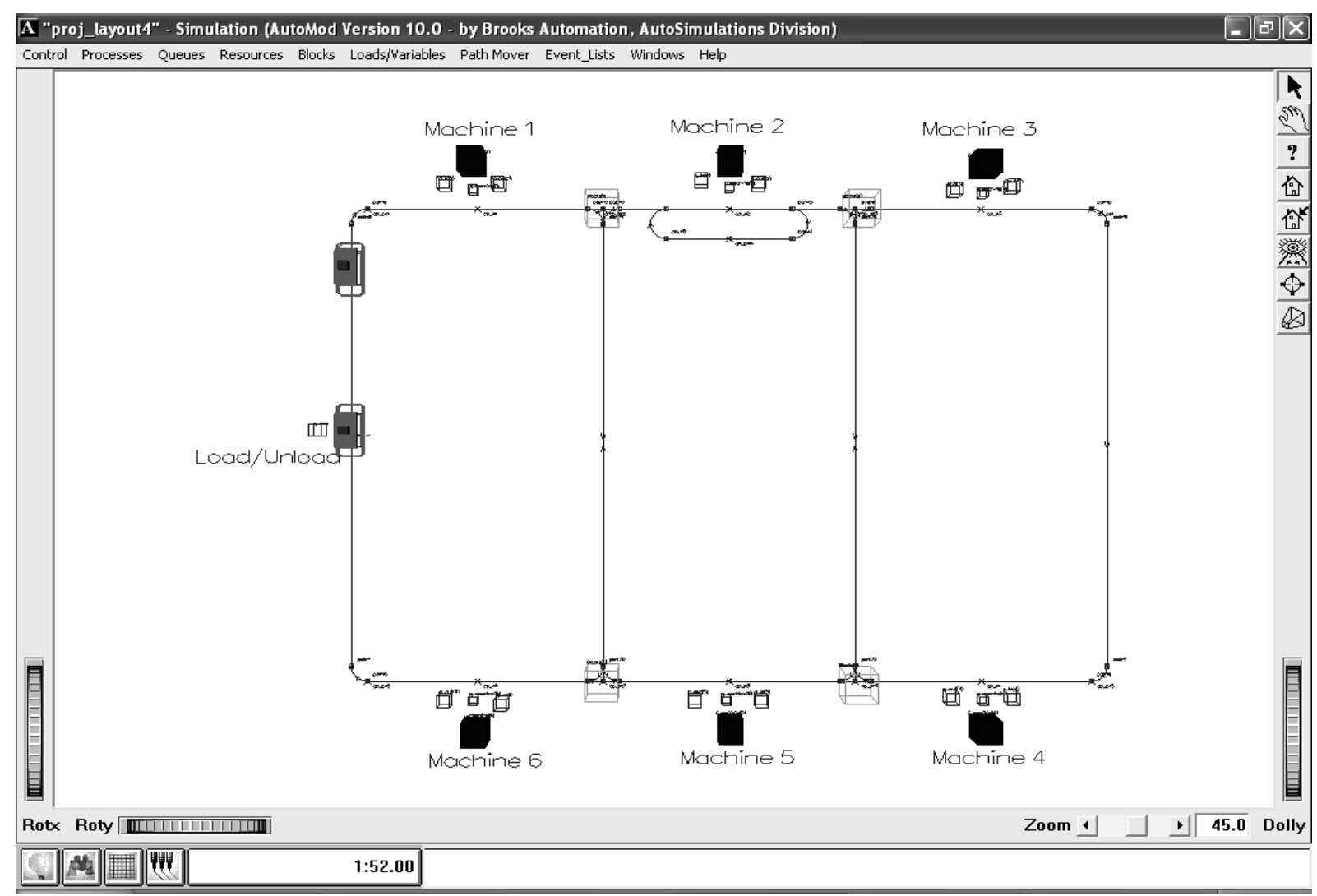

Figure 4: FMS Layout 3. 
Reddy, Rao: Flexible Manufacturing Systems Modelling and Performance Evaluation ...

Table IX: Travel time Matrix 2.

Table X: Travel time Matrix 3.

\begin{tabular}{|c|c|c|c|c|c|c|}
\hline & $\mathrm{L} / \mathrm{U}$ & $\mathrm{M}_{1}$ & $\mathrm{M}_{2}$ & $\mathrm{M}_{3}$ & $\mathrm{M}_{4}$ & $\mathrm{M}_{5}$ \\
\hline $\mathrm{L} / \mathrm{U}$ & 0 & 2.5 & 4.5 & 4.5 & 6.5 & 5.5 \\
\hline $\mathrm{M}_{1}$ & 4.5 & 0 & 3.5 & 3.5 & 5.5 & 4.5 \\
\hline $\mathrm{M}_{2}$ & 2.5 & 3.5 & 0 & 5.5 & 7.5 & 6.5 \\
\hline $\mathrm{M}_{3}$ & 6.5 & 7.5 & 5.5 & 0 & 3.5 & 2.5 \\
\hline $\mathrm{M}_{4}$ & 4.5 & 5.5 & 3.5 & 3.5 & 0 & 4.5 \\
\hline $\mathrm{M}_{5}$ & 5.5 & 6.5 & 4.5 & 4.5 & 2.5 & 0 \\
\hline
\end{tabular}

\begin{tabular}{|c|c|c|c|c|c|c|c|}
\hline & $\mathrm{L} / \mathrm{U}$ & $\mathrm{M}_{1}$ & $\mathrm{M}_{2}$ & $\mathrm{M}_{3}$ & $\mathrm{M}_{4}$ & $\mathrm{M}_{5}$ & $\mathrm{M}_{6}$ \\
\hline $\mathrm{L} / \mathrm{U}$ & 0 & 4 & 6 & 8 & 14 & 12 & 10 \\
\hline $\mathrm{M}_{1}$ & 10 & 0 & 3 & 5 & 11 & 9 & 7 \\
\hline $\mathrm{M}_{2}$ & 12 & 15 & 0 & 3 & 9 & 7 & 9 \\
\hline $\mathrm{M}_{3}$ & 14 & 17 & 15 & 0 & 7 & 9 & 11 \\
\hline $\mathrm{M}_{4}$ & 8 & 11 & 9 & 7 & 0 & 3 & 5 \\
\hline $\mathrm{M}_{5}$ & 6 & 9 & 7 & 9 & 15 & 0 & 3 \\
\hline $\mathrm{M}_{6}$ & 4 & 7 & 9 & 11 & 17 & 15 & 0 \\
\hline
\end{tabular}

Table XI: Simulation results of large size problems (Makespan).

\begin{tabular}{|c|c|c|c|c|c|c|c|c|c|}
\hline \multirow{2}{*}{$\begin{array}{c}\text { Layout/ } \\
\text { Job set } \\
\text { details }\end{array}$} & \multicolumn{3}{|c|}{ Job dispatching rule } \\
\cline { 2 - 11 } & \multicolumn{3}{|c|}{ FCFS } & \multicolumn{7}{c|}{ SPT } & \multicolumn{3}{c|}{ LPT } \\
\cline { 2 - 11 } & At & Closest & Oldest & At & Closest & Oldest & At & Closest & Oldest \\
\hline L1 J1 & 887.252 & 877.997 & 803.514 & 962.230 & 853.552 & 734.806 & 746.740 & 731.370 & 830.855 \\
\hline L1 J2 & 1062.100 & 1028.452 & 1072.410 & 1044.368 & 1049.436 & 953.401 & 987.421 & 987.421 & 1097.731 \\
\hline L1 J3 & 1650.218 & 1605.218 & 1561.697 & 1460.341 & 1466.607 & 1633.645 & 1610.863 & 1610.863 & 1513.984 \\
\hline L2 J1 & 917.738 & 837.698 & 812.951 & 913.738 & 913.738 & 716.487 & 705.544 & 705.544 & 807.332 \\
\hline L2 J2 & 970.897 & 961.794 & 1053.266 & 1036.004 & 1044.459 & 939.550 & 985.175 & 981.045 & 1078.993 \\
\hline L2 J3 & 1606.004 & 1606.004 & 1561.586 & 1527.197 & 1550.839 & 1729.123 & 1678.016 & 1678.016 & 1604.176 \\
\hline L3 J4 & 219.830 & 206.849 & 225.264 & 235.368 & 222.041 & 231.109 & 241.335 & 206.312 & 227.115 \\
\hline L3 J5 & 223.835 & 282.854 & 233.401 & 275.205 & 253.253 & 277.972 & 241.738 & 268.500 & 243.511 \\
\hline
\end{tabular}

J1: Job Set 1 - Lawrence [21], 10×5

(10 Jobs each with 5 operations, 5 Machines Problem)

J2: Job Set 2 - Lawrence [21], 15×5

(15 Jobs each with 5 operations, 5 Machines Problem)

J3: Job Set $3-$ Fisher and Thompson [20], 20×5

(20 Jobs each with 5 operations, 5 Machines Problem)

J4: Job Set 4 - Fisher and Thompson [20], 6×6

(6 Jobs each with 6 operations, 6 Machines Problem)

J5: Job Set 5 - Fisher and Thompson [20], 7×6

(7 Jobs each with 6 operations, 6 Machines Problem)

L1: FMS Layout 1 - 5 Machines only uni-directional flow of jobs

L2: FMS Layout 2 - 5 Machines bi-directional flow of jobs

L3: FMS Layout 3 - 6 Machines bi-directional flow ladder layout 


\section{CONCLUSIONS}

The purpose of this study is to model a flexible manufacturing system using Automod and to consider simultaneously both machine and vehicle scheduling to minimise the makespan.

Design and operational issues of automated material handling system such as number of vehicles, vehicle dispatching rules on different FMS layouts are studied.

It is observed that the "At" vehicle assignment rule is performing better in most of the test cases as it can pick the job immediately.

It is observed that the performance of bi-directional paths are better in case of mixed nature of machine sequence and its performance is found to be better for FCFS rule.

It is observed that uni-directional paths are better in case of uni-directional machine sequence and its performance is found to be better for LPT rule.

It is found that in most of the test cases the vehicle utilization is found to be better in case of 2 vehicles.

The vehicle congestion problems are found to be more when the number is above 2 and for a single vehicle case there is a noticeable increase in the makespan.

\section{SCOPE FOR FUTURE WORK}

The present study is limited to maximum of 100 operations and processing time based rules however can be extended for large size problems for different operation schedules and the simulation software can be integrated with FMS environments using SDX files.

\section{REFERENCES}

[1] Egbelu, P. J.; Tanchoco, J. M. A. (1984). Characterization of automated guided vehicle dispatching rules, International Journal of Production Research, Vol. 22, No. 3, 359-374

[2] Mahadevan, B.; Narendran, T. T. (1990). Design of an automated guided vehicle-based material handling system for a flexible manufacturing system, International Journal of Production Research, Vol. 28, No. 9, 1611-1622

[3] Raju, R. K.; Chetty, K. O. V. (1993). Priority nets for scheduling flexible manufacturing systems, Journal of manufacturing systems, Vol. 12, No. 4, 326-340

[4] Kim, M. H.; Kim, Y. D. (1994). Simulation-based real-time scheduling in a flexible manufacturing system, Journal of manufacturing systems, Vol. 13, No. 2, 85-93

[5] Drake, G.; Smith, J. S. (1996). Simulation system for real time planning, scheduling and control, Proceedings of Winter Simulation Conference, 1083-1090

[6] Ulusoy, G.; Serifoglu, F. S.; Bilge, U. (1997). A genetic algorithm approach to the simultaneous scheduling of machines and automated guided vehicles, Computers \& Operations Research, Vol. 24, No. 4, 335-351

[7] Sabuncuoglu, I. (1998). A study of scheduling rules of FMS: A simulation approach, International Journal of Production Research, Vol. 36, No. 2, 527-546

[8] Anwar, M. F.; Nagi, R. (1998). Integrated scheduling of material handling and manufacturing activities for JIT production of complex assemblies, International Journal of Production Research, Vol. 36, No. 3, 653-681

[9] Ozden, M. (1998). A simulation study of multiple load carrying automated guided vehicles in a flexible manufacturing system, International Journal of Production Research, Vol. 26, No. 8, 1353-1366

[10] Rajotia, S.; Shanker, K.; Batra, J. L. (1998). Heuristics for configuring a mixed uni/bi-directional flow path for an AGV system, International Journal of Production Research, Vol. 36, No. 7, 1779-1799 
[11] Paprotny, I.; Zhao, W.; Mackulak, G. T. (1999). Reducing model creation cycle time by automated conversion of a CAD AMHS layout design, Proceedings of the Winter Simulation Conference, 779-783

[12] Schulz, M.; Stanley, T. D.; Renelt, B.; Sturm, R.; Schwertschlager, O. (2000). Simulation based decision support for future $300 \mathrm{~mm}$ automated material handling, Proceedings of the Winter Simulation Conference, 1518-1522

[13] Rohrer, M. W. (2000). Automod tutorial, Proceedings of the Winter Simulation Conference, 170176

[14] Sly, D.; Moorthy, S. (2001). Simulation data exchange (SDX) implementations and use, Proceedings of the Winter Simulation Conference, 1473-1477

[15] Fritz, R.; Horvath, S.; Orellana, C.; Wohlers, J.; White, K. P.; Fairbrother, R.; Terry, W. (2002). Real- time control of a discrete-event simulation using an external controller: a feasibility study with application to mail distribution systems, IEEE Systems Engineering and Information Engineering Design Symposium, Virginia

[16] Smith, J. S. (2003). Survey on the use of simulation for manufacturing system design and operation, Journal of manufacturing systems, Vol. 22, No. 2, 157-171

[17] Reddy, B. S. P.; Rao, C. S. P. (2006). A hybrid multi-objective GA for simultaneous scheduling of machines and AGVs in FMS, International Journal of Advanced Manufacturing Technology, Vol. 31, No. 5-6, 602-613

[18] Haijun, M.; Hongtao, G.; Chenlin, M.; Xuhong, L.; Jie, H. (2008). Modeling and analysis of steel logistics center based Automod simulation platform, Journal of Southeast University (Natural Science Edition), Vol. 38, No. 2

[19] Chenglin, M.; Haijun, M.; Xuhong, L.; Wensi, S. (2009). Modeling and analysis of integrated logistics park based Automod simulation platform, International Conference on Intelligent Computation Technology and Automation, Vol. 2, 86-89

[20] Fisher, H.; Thompson, G. L. (1963). Probabilistic learning combinations of local job-shop scheduling rules, in Industrial Scheduling, Prentice Hall, Englewood Cliffs, 225-251

[21] Lawrence, S. (1984). Resource constrained project scheduling: an experimental investigation of heuristic scheduling techniques (Supplement), Technical Report, Graduate School of Industrial Administration, Carnegie-Mellon University, Pittsburgh, Pennsylvania 